\title{
A 3T Phase-Sensitive Inversion Recovery MRI Sequence Improves Detection of Cervical Spinal Cord Lesions and Shows Active Lesions in Patients with Multiple Sclerosis
}

\author{
(D) A. Fechner, (D). Savatovsky, (D). El Methni, (D).C. Sadik, (D) O. Gout, (D) R. Deschamps, (D)A. Gueguen, and (D)A. Lecler
}

\begin{abstract}
BACKGROUND AND PURPOSE: Magnetic Resonance Imaging is the modality of choice to detect spinal cord lesions in patients with Multiple Sclerosis (MS). However, this imaging is challenging. New sequences such as phase-sensitive inversion recovery have been developed to improve detection. Our aim was to compare a 3D phase-sensitive inversion recovery and a conventional imaging dataset including postcontrast T2WI and TIWI to detect MS spinal cord lesions.
\end{abstract}

MATERIALS AND METHODS: This retrospective single-center study included 100 consecutive patients with MS (mean age, 41 years) from January 2015 to June 2016. One senior neuroradiologist and 1 junior radiologist blinded to clinical data checked for new spinal cord lesions, individually analyzing conventional and 3D phase-sensitive inversion recovery datasets separately, placing a 3-week delay between the 2 readings. A consensus reading was done with a third senior neuroradiologist. A Wilcoxon test was used to compare the 2 imaging datasets. Intra- and interobserver agreement was assessed by the $\kappa$ coefficient.

RESULTS: 3D phase-sensitive inversion recovery detected significantly more lesions than conventional imaging (480 versus $168, P<.001)$. Eleven patients had no detected lesions on T2WI, whereas 3D phase-sensitive inversion recovery detected at least 1 lesion. All postcontrast TTWI enhancing lesions were also visible on 3D phase-sensitive inversion recovery. The signal-to-noise ratio was significantly higher using $3 \mathrm{D}$ phase-sensitive inversion recovery $(0.63$ versus $0.46, P=.03)$. Mean reading confidence was significantly higher using 3D phase-sensitive inversion recovery. Inter- and intraobserver agreement was good for both datasets.

CONCLUSIONS: Our study showed that 3D phase-sensitive inversion recovery significantly improved detection of cervical spinal cord lesions, including both enhancing and nonenhancing lesions in patients with MS.

ABBREVIATIONS: CNR = contrast-to-noise ratio; PSIR = phase-sensitive inversion recovery; MAGNIMS = Magnetic Resonance Imaging in Multiple Sclerosis; MERGE = Multi-Echo Recombined Gradient Echo; PSTT-IR = phase-sensitive T1-weighted inversion-recovery MR imaging

M ultiple sclerosis is a neurodegenerative autoimmune demyelinating disease affecting the central nervous system, primarily involving the brain and the spinal cord. Imaging of the spinal cord is recommended because these results can detect silent lesions, described in $30 \%-40 \%$ of patients with radiologically or clinically isolated syndrome. ${ }^{1}$ Spinal imaging defines lesion dissemination in spatial terms, thus increasing accurate MS diagnoses according to the Magnetic Resonance Imaging in MS

Received September 17, 2018; accepted after revision November 29. From the Departments of Neuroradiology (A.F., J.S., J.C.S., A.L.) and Neurology (O.G., R.D., A.G.), Fondation Ophtalmologique Adolphe de Rothschild, Paris, France; and Laboratoire MAP5, UMR CNRS 8145 (J.E.M.), Université Paris Descartes, Paris, France.

Please address correspondence to Augustin Lecler, MD, PhD, Department of Neuroradiology, Fondation Ophtalmologique Adolphe de Rothschild, 25 rue Manin, 75019 Paris, France; e-mail: alecler@for.paris

$\square$ Indicates article with supplemental on-line photos.

http://dx.doi.org/10.3174/ajnr.A5941
(MAGNIMS) and McDonald criteria, ${ }^{2,3}$ once differential diagnoses have been excluded. Imaging also helps predict management decisions because high spinal cord lesion load is linked to poorer prognosis. ${ }^{4}$

MR imaging is the technique of choice to fully visualize the spinal cord. ${ }^{5}$ This type of imaging proves challenging due to many visual artifacts generated from this area caused by cardiac pulsations, breathing, and CSF movement. ${ }^{6-9}$ Spinal cord lesions are classically studied with sagittal and axial T2-weighted sequences and postcontrast T1-weighted sequences, but these sequences were reported to lack sensitivity during the search of MS lesions.

To overcome challenges with spine imaging and increase both the sensitivity and specificity to detect inflammatory medullary lesions, the literature indicates a few other reliable MR imaging sequences, such as proton-density imaging, ${ }^{10}$ STIR, ${ }^{11,12}$ phasesensitive T1-weighted inversion-recovery MR imaging (PST1IR) ${ }^{13}$ Multi-Echo Recombined Gradient Echo (MERGE; GE Healthcare, Milwaukee, Wisconsin), ${ }^{14}$ white matter-suppressed 
T1 inversion recovery, ${ }^{15}$ double inversion recovery, ${ }^{16}$ and MPRAGE. ${ }^{17}$ Recent studies have shown that the phase-sensitive inversion recovery (PSIR) sequence, consisting of a heavily T1weighted inversion recovery combined with phase-sensitive reconstruction, ${ }^{13,18}$ had the highest lesion-to-cord contrast and lesion-limit definition. ${ }^{13}$ Therefore, this sequence seemed particularly promising for the detection of spinal cord lesions.

Our center developed a 3D-PSIR sequence at 3T for the spine. The aim of this study was to evaluate its sensitivity to detect spinal cord lesions in patients with MS compared with a conventional set including postcontrast T2WI and T1WI sequences.

\section{MATERIALS AND METHODS \\ Study Design}

We conducted a retrospective systematic chart review in a tertiary referral center specializing in neurologic diseases, the Rothschild Ophthalmological Foundation, Paris, France. This study was approved by our institutional research ethics board and adhered to the tenets of the Declaration of Helsinki. This study follows the Strengthening the Reporting of Observational Studies in Epidemiology guidelines. ${ }^{19}$

\section{Patients}

From January 2015 to June 2016, one hundred consecutive patients were included. Inclusion criteria were the following: 1) older than 18 years of age; 2) a confirmed diagnosis of multiple sclerosis using the 2010 criteria for $\mathrm{MS}^{3}$; 3 ) the presence of spinal cord MR imaging, including 1 conventional set of sagittal T2- and postcontrast T1-weighted imaging and one 3D-PSIR sequence.

\section{Clinical Charts}

All patients' medical charts were systematically reviewed to retrieve clinical data such as the type of MS, demographics, and the score from the Expanded Disability Status Scale. ${ }^{20}$

\section{MR Imaging}

All MR imaging examinations were performed with the same 3T Ingenia scanner (Philips Healthcare, Best, the Netherlands) with a 16-channel head coil and a posterior spine coil. Two sets of images were acquired during the same examination. The first one was considered a conventional set, including sagittal T2 (TR, $2805 \mathrm{~ms}$; TE, $100 \mathrm{~ms}$; number of excitations, 2; slice thickness, $2 \mathrm{~mm}$ with no gap; FOV, $360 \times 360 \mathrm{~mm}$; bandwidth, $773 \mathrm{~Hz}$; acquisition matrix, $376 \times 297$; acquisition duration, 3 minutes and $48 \mathrm{sec}-$ onds) and sagittal T1WI (TR, $512 \mathrm{~ms}$; TE, $16 \mathrm{~ms}$; number of excitations, 1 ; slice thickness, $3 \mathrm{~mm}$ with no gap; FOV, $360 \times 360$ mm; bandwidth, $260 \mathrm{~Hz}$; acquisition matrix, $328 \times 272$; acquisition duration, 2 minutes and 6 seconds), as recommended by the MAGNIMS consensus guidelines. ${ }^{2}$ The second one, a 3D-PSIR set, included 2 magnitude images and 1 phase-corrected real image automatically provided from a unique optimized 3D-PSIR acquisition (scan mode 3D; sagittal native acquisition plane; technique, fast-field echo; TR, $5.8 \mathrm{~ms}$; TE, $2.6 \mathrm{~ms}$; number of excitations, 1 ; slice thickness, $1 \mathrm{~mm}$ with no gap; FOV, $320 \times 220 \mathrm{~mm}$; bandwidth, $362 \mathrm{~Hz}$; acquisition matrix, $320 \times 220$; voxel size, $1.0 \times 1.0 \times 1.0 \mathrm{~mm}$; turbo field echo factor, 69 ; turbo field echo prepulse inversion time, $350 \mathrm{~ms}$; turbo field echo shots, 79; turbo field echo shot duration, $400 \mathrm{~ms}$; turbo field echo shot interval, $758 \mathrm{~ms}$; flip angle, $15^{\circ}$; fat suppression with spectral presaturation with inversion recovery; acquisition duration, 4 minutes and 6 seconds) (On-line Figs 1 and 2). The first sequences were acquired 10 minutes after a single bolus $(0.1 \mathrm{mmol} / \mathrm{kg})$ of gadobutrol (Gadovist; Bayer Schering Pharma, Berlin, Germany). The conventional set sequences were always acquired before the $3 \mathrm{D}$-PSIR.

\section{Image Analysis}

Two radiologists, blinded to clinical data, individually read the randomized results of the conventional set and those of the $3 \mathrm{D}$ PSIR reformatted sequence in the sagittal plane; 3 weeks or more passed between the 2 readings. The first senior neuroradiologist was specialized in neuroimaging with 8 years of experience (A.L.), and the second was a junior radiologist with no experience in neuroimaging (A.F.). Six weeks later, a criterion standard consensus reading session was performed by the third reader, a second senior neuroradiologist with 20 years of experience (J.S.), also blinded to clinical data. At the end of this last consensus session, readers eventually looked at the entire imaging dataset, including all planes of the 3D-PSIR or axial T2WI if performed to determine whether the lesions observed in 3D-PSIR were false-positive. A final reading session was performed 6 weeks later to analyze intraobserver concordance. All reading sessions were performed on a dedicated workstation with the Carestream Vue PACS software.

The readers assessed the following characteristics of patients' MR imaging:

- The primary judgment criterion was the presence and number of spinal cord lesions, defined as hyperintense lesions on T2WI and hypo- or hyperintense lesions on the phase-corrected real image or magnitude images of the 3D-PSIR, respectively (Online Fig 1). To avoid overestimation, readers were instructed not to count lesions in areas too full of artifacts and to report only evident and well-delineated lesions on the 3D-PSIR sequence.

- The precise level of the lesions in the sagittal plane, according to the related cervical level.

- The precise location of the lesions in the axial plane, defined as central or peripheral, and the length of the lesions in the sagittal plane.

- The confidence in detecting spinal cord lesions, was measured as follows: 1 corresponded to low confidence; 2, moderate-tohigh confidence; and 3, very high confidence.

- The presence of active lesions defined as an enhancement on the postcontrast T1WI or a high signal intensity on both magnitude and phase-corrected real 3D-PSIR images.

Quantitative measurements of MR imaging signals were obtained by drawing 3 ROIs per patient: in the largest spinal cord lesion (lesion signal) and in the normal-appearing spinal cord (cord signal) and background (background signal).

The contrast-to-noise ratio (CNR) was calculated according to the following formula: CNR $=($ Lesion Signal - Cord Signal $) /$ Noise SD.

The SNR was calculated according to the following formula: $\mathrm{SNR}=($ Lesion Signal - Background Signal $) /$ Noise SD.

AJNR Am J Neuroradiol 40:370-75 Feb 2019 www.ajnr.org 371 


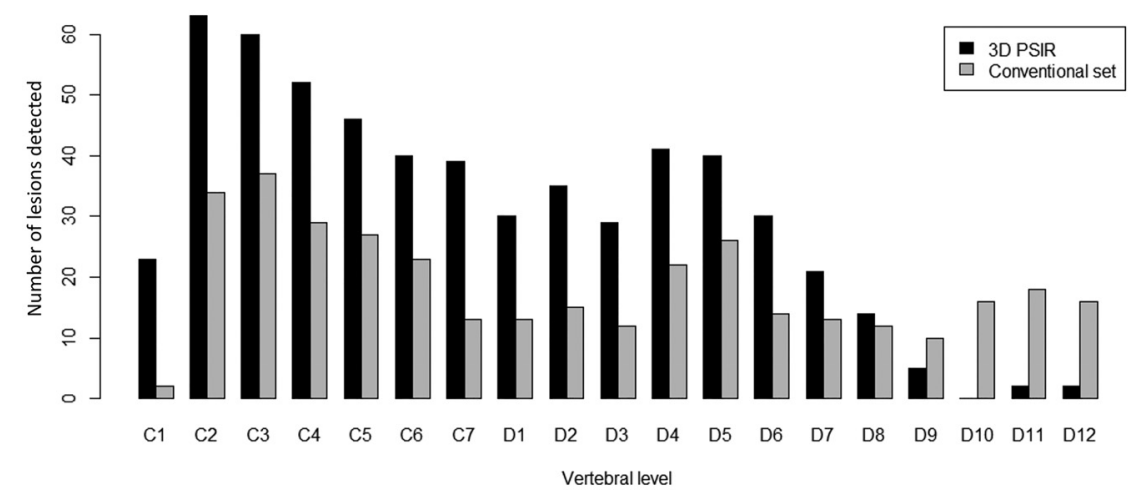

FIG 1. Comparison of the number and distribution of MS lesions detected in the spinal cord using the conventional set (gray) compared with 3D-PSIR (black) imaging.
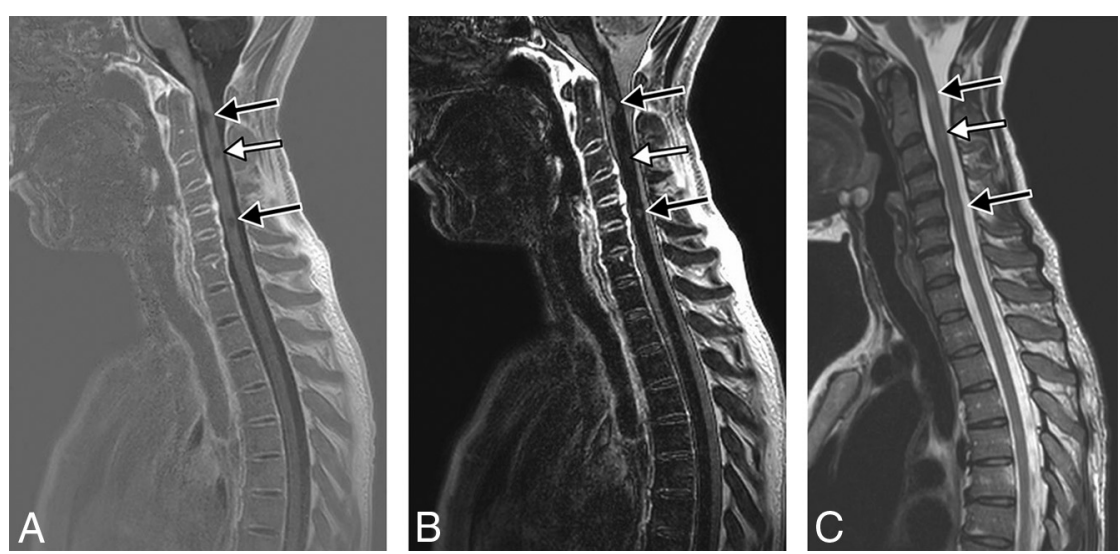

FIG 2. Follow-up MR imaging of a 32-year-old woman with relapsing-remitting MS. 3D-PSIR reformatted in the sagittal plane shows 2 conspicuous (black arrows) and 1 less obvious (white arrow) cervical spinal cord lesions seen as hypointense on the phase-corrected real image $(A)$ and hyperintense on the magnitude image $(B)$. The same lesions are all difficult to see on the sagittal T2WI (C).

\section{Statistical Analysis}

Quantitative variables were presented as mean (SD) and median (interquartile range); and categoric variables, as percentages. A Wilcoxon test was used to compare the number of lesions detected between both datasets as well as the readers' confidence and quantitative CNR and SNR values. Inter- and intraobserver agreement for the MR imaging reading was assessed using a nonweighted Cohen $\kappa$ statistic and was interpreted as follows: $0.0-0.2$, poor correlation; 0.21-0.4, fair correlation; 0.41-0.6, moderate correlation; $0.61-0.8$, good correlation; and $0.81-1$, almost perfect correlation. ${ }^{21}$ A $P$ value $<.05$ was considered statistically significant. Data were analyzed using the $\mathrm{R}$ software package. ${ }^{22}$

\section{RESULTS}

\section{Demographic and Clinical Characteristics}

One hundred consecutive patients with MS were included (55 women and 45 men; mean age, 41 years; 82 with relapsing-remitting MS, 12 with secondary-progressive MS, and 6 with primaryprogressive MS). The mean Expanded Disability Status Scale score was $3.2 \pm 2.1$. The mean disease duration was $13.6 \pm 9.2$ years.

\section{Spinal Cord Lesions}

Of 100 patients, 67 had at least 1 spinal cord lesion, and 10 had an active enhancing lesion, found using both MR imaging methods under investigation. Lesions were more likely to be located on the periphery of the spinal cord than centrally: $379(79 \%)$ versus $101(21 \%)$. There was no statistical difference in the measurement of the mean length between the 2 imaging sets: 11.44 versus $10.23 \mathrm{~mm}$ using 3D-PSIR or the conventional set, respectively.

\section{Detection of Lesions}

3D-PSIR detected significantly more overall lesions than the conventional set (480 versus 168 , respectively, $P=6 \times$ $\left.10^{-13}\right)$. 3D-PSIR also detected significantly more lesions in both cervical (270 versus $\left.83, P=9 \times 10^{-6}\right)$ and dorsal regions (210 versus $85, P=2 \times 10^{-4}$ ) (Figs 1 and 2). Eleven patients had at least 1 lesion on 3D-PSIR images with no lesion detected on the conventional set (Fig 3). None of these lesions were considered false-positive. All lesions detected with the conventional set above T10 were also visible on 3D-PSIR. All enhancing lesions were visible on both 3D-PSIR and the conventional set (Fig 4).

Confidence in lesion detection was significantly higher with 3D-PSIR than with the conventional set (mean confidence, 2.6 versus $2.2 ; P=3 \times 10^{-11}$ ), with $95 \%$ of lesions detected with a moderate-to-very-high degree of confidence, and $67 \%$, with a very high degree of confidence on $3 \mathrm{D}$ PSIR versus $87 \%$ and $50 \%$ on the conventional set, respectively.

\section{CNR and SNR Evaluation}

Both CNR and SNR were significantly higher with 3D-PSIR than with the conventional set (mean, 0.48 versus 0.26 ; $P<1 \times 10^{-5}$; and 0.63 versus $0.46, P=.03$, respectively).

\section{Interreader Concordance}

Overall interreader concordance was good $(\kappa=0.7)$ without any differences between the 3D-PSIR and the conventional set $(\kappa=$ 0.7 and 0.8 , respectively).

Overall intrareader concordance was good $(\kappa=0.7$ and 0.8 for readers 1 and 2, respectively), without any differences between the 3D-PSIR and the conventional set.

\section{DISCUSSION}

Our study showed that 3D-PSIR improved overall spinal cord lesion detection in patients with MS, with higher reader-reported confidence and SNR, showing a substantial number of lesions not seen with a conventional imaging set and active enhancing lesions.

Our results are consistent with those in previous studies evaluating new sequences that overcome the challenges of spine im- 


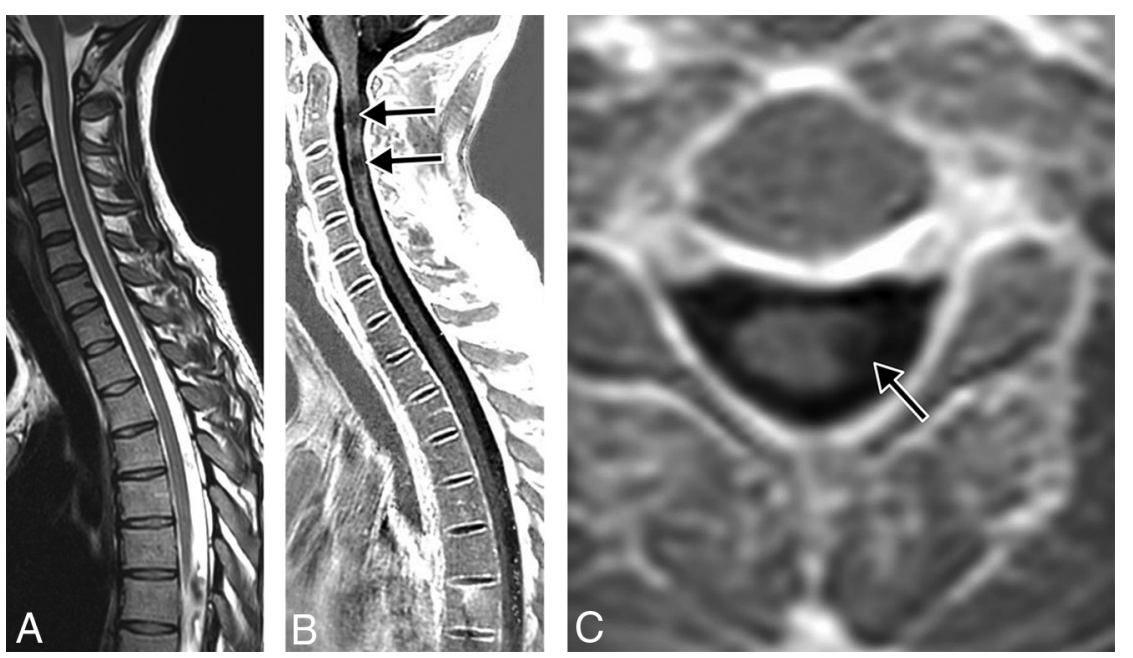

FIG 3. Follow-up MR imaging of a 40-year-old woman with secondary-progressive MS. Sagittal T2WI (A) shows no spinal cord lesions. 3D-PSIR reformatted in the sagittal plane $(B)$ demonstrates 2 cervical spinal cord lesions (black arrows). 3D-PSIR reformatted in the axial plane (C) provides accurate localization of the lesions (black arrow).

aging in MS by increasing both the sensitivity and specificity of the MR imaging, such as proton-density imaging, ${ }^{10}$ STIR,,${ }^{11,12}$ PST1IR, ${ }^{13}$ MERGE, ${ }^{14}$ double inversion recovery, ${ }^{16}$ and MPRAGE. ${ }^{17}$ We reported significantly higher detection of spinal cord lesions with 3D-PSIR, with $65 \%$ of overall lesions not visible on the conventional set, which is consistent with the $28 \%-72 \%$ increased detection rate using the optimized spinal cord sequences in the literature. ${ }^{10,12,14,16,23,24}$ Eleven (11\%) patients without any lesions detected with the conventional set had at least 1 lesion captured using 3D-PSIR, which is similar to the $3 \%-24 \%$ previously reported. ${ }^{10,12,16}$

One unreported-yet-major interest of 3D-PSIR was its ability to detect enhancement of active spinal cord lesions with similar detection rates compared with postcontrast $\mathrm{T} 1$ sequences. 3DPSIR is derived from a T1WI sequence, thus making it sensitive to contrast injection. ${ }^{13,18}$ This finding means that a unique "all-inone" 3D-PSIR sequence might be sufficient to provide data on both the spinal cord lesion burden and its activity at the same time, reflecting the dissemination in space and time of the McDonald criteria and supporting accurate monitoring of treatment efficacy. ${ }^{25}$ However, only 10 patients had enhancing lesions; thus, our sample was too small to draw any firm conclusions regarding the detectability of enhancement with the 3D-PSIR.

Spinal cord imaging remains challenging because the targeted surface area on the body is small in a region prone to numerous imaging artifacts due to respiration, cardiac contractions, partial volumes, or CSF pulsations..$^{6-9}$ Our study demonstrated that the use of a high-resolution 3D-PSIR sequence helped overcome some of these problems and that analyzing data in 3 different planes with 3D-PSIR enabled a higher lesion count, a better delineation and localization of the lesions, and exclusion of equivocal abnormalities, as reported by studies evaluating the clinical interest of biplanar or 3D MR imaging. ${ }^{16,17,24}$ We showed that the confidence in detection and lesion conspicuity was significantly higher with 3D-PSIR as opposed to the conventional set, which may be explained by the increased contrast of both CNR and SNR, as reported in the literature for 2D-PSIR sequences. ${ }^{12,13,18,26}$ On visual inspection, 3D-PSIR sequences subjectively provided a markedly higher lesion-to-cord contrast and signal-tonoise ratio so that readers were more confident and comfortable making clinical judgements.

Improving detection of spinal cord lesions is crucial for patients facing a potential diagnosis of MS. Clearly identified lesions fulfill the McDonald criteria for early and definitive diagnosis, ${ }^{3}$ and they are highly predictive of conversion to MS in patients with clinically or radiologically isolated symptoms. ${ }^{1,27}$ Quantification of disease activity is important for monitoring treatment efficacy. ${ }^{25}$ Therefore, recent US Consortium of Multiple Sclerosis Centers and MAGNIMS consensus guidelines modified their recommendations for performing the most sensitive spinal cord MR imaging protocol, using at least $2 \mathrm{MR}$ images such as T2 and short $\mathrm{T} 1$ inversion recovery, $\mathrm{T} 2$ and double inversion recovery, $\mathrm{T} 2$ and postcontrast $\mathrm{T} 1$ sequences, ${ }^{28}$ or a combination of either T2 and short $\mathrm{T} 1$ inversion recovery, $\mathrm{T} 2$ and double inversion recovery, or T2 and postcontrast T1 sequences, ${ }^{2}$ respectively. The advantages of 3D-PSIR related to the above suggest that a single 3D-PSIR sequence could be a potential replacement strategy and should at least be considered as a standard spinal cord sequence in patients with MS.

In our study, the duration of the 3D-PSIR was almost the same as that of the sagittal T2WI (15 seconds longer) and was almost 2 minutes faster than the conventional imaging set (4 minutes and 54 seconds faster). Reformatted images in the axial plane would serve as an advantageous replacement of an optional supplementary conventional axial T2WI by reducing the acquisition time (the duration of the axial T2WI was 3 minutes and 12 seconds in our center; thus, the overall saved time could reach 8 minutes and 6 seconds) and would provide greater spinal cord coverage. Moreover, the new guidelines suggest that performing brain and cervical cord MR imaging at the same time would be advantageous for the diagnostic evaluation of patients with or without transverse myelitis and would reduce the number of patients requiring subsequent MR imaging appointments. ${ }^{28}$

Decreasing gadolinium injections by simultaneously performing brain and spinal cord MR imaging using a unique contrast injection of gadolinium could help with patient safety for the long term. We support this approach in these often young patients because they will have a high number of MRIs during their lifetimes and thus receive high doses of contrast injections, which can negatively affect their safety because the product might accumulate in their brains. ${ }^{29-31}$ However, combining the brain and spine MR imaging is time-consuming and difficult to establish in current practice. The addition of a single 3D-PSIR covering the spinal cord to the brain MR imaging would therefore be a good compromise between a reasonable MR imaging duration time (4 minutes longer) and the accurate evaluation of MS. 


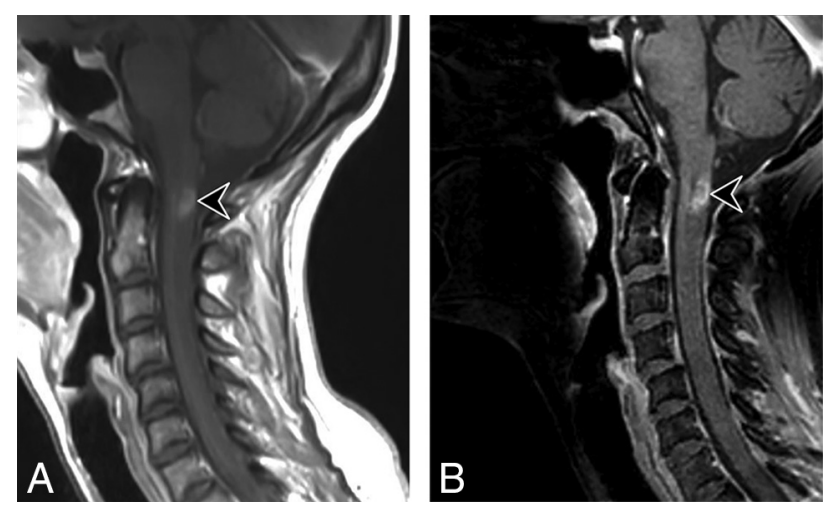

FIG 4. Follow-up of a 32-year-old woman with relapsing-remitting MS presenting with acute paresthesia of the upper arm. Sagittal postcontrast TIWI $(A)$ shows an enhancing cervical spinal cord lesion (black arrowhead) corresponding to an active inflammatory lesion. 3D-PSIR reformatted in the sagittal plane shows the same enhancing lesion on the magnitude image $(B)$.

Our study has several limitations. First, although this was a relatively large cohort in comparison with previous studies evaluating optimized spinal cord MR imaging sequences, the overall number of patients is low in a single center.

Second, our 3D-PSIR did not cover the entire spinal cord, but only the cervical region down to two-thirds of the dorsal region, whereas the conventional set analyzed the whole spinal cord. This coverage could have provided an underestimation of lesions detected with 3D-PSIR. The MR imaging signal decreased at the lower part of the spinal cord and levels under T9 were not correctly visualized. However, current recommendations indicate that coverage should include the cervical cord at a minimum because most spinal cord lesions in MS involve the cervical cord ${ }^{32}$ and that the routine examination of the thoracic cord is not necessary unless there are clinical symptoms at that level. ${ }^{28}$

Third, we compared 3D-PSIR with 1-mm slices with a conventional set of postcontrast 2D T2WI and T1WI with 2- to 3-mm thickness; thus, the higher detection rate of the 3D-PSIR might be due, in part, to the higher resolution or the $3 \mathrm{D}$ acquisitions. We tried to minimize the $3 \mathrm{D}$ effect by reading only the $2 \mathrm{D}$ sagittal planes of the 3D-PSIR. We also did not compare the PSIR with a STIR sequence, which has a higher sensitivity compared with T2WI. However, this institutional practice was in accord with the last US Consortium of Multiple Sclerosis Centers and European MAGNIMS consensus guidelines, ${ }^{2,28}$ and conventional T1 and T2 sequences remain the most commonly used for spinal cord MS lesion detection in clinical institutions. ${ }^{33}$

Fourth, we always performed the conventional set sequences before the 3D-PSIR, so the postgadolinium impregnation time was longer for 3D-PSIR, which could potentially have had an impact on the detection of spine lesions. However, the 10-minute delay before gadolinium injection and the image acquisitions is supposed to have minimized this effect.

Fifth, readers knew which method they were assessing, which could have led to a certain bias.

Finally, we did not correlate the results with pathology specimens and therefore cannot be sure that all lesions detected were, in fact, MS lesions. However, nonspecific white matter lesions are reported to be extremely uncommon in the spinal cord, in con- trast to their frequent occurrence in the brain, ${ }^{34}$ and it was impossible to have a real standard of reference because this would imply postmortem histologic examinations. Moreover, there are good histopathologic data to support the accuracy of optimized spinal cord sequences. ${ }^{35}$

\section{CONCLUSIONS}

Our study showed that 3D-PSIR improved overall spinal cord lesion detection in patients with MS, with higher reader-reported confidence and SNR, showing a substantial number of lesions not seen with a conventional imaging set and active enhancing lesions. Further studies would be helpful to evaluate prospectively the value of the imaging method in comparison with other new MR images.

\section{ACKNOWLEDGMENT}

Laura McMaster provided professional English-language medical editing of this article.

Disclosures: Alix Fechner-UNRELATED: Employment: Fondation Ophtalmologique de Rothschild. Roman Deschamps—UNRELATED: Support for Travel to Meetings for the Study or Other Purposes: Biogen. Antoine Gueguen-UNRELATED: Board Membership: Roche, Merck, Mylan, Novartis; Consultancy: Mylan, Novartis; Payment for Lectures Including Service on Speakers Bureaus: Teva Pharmaceutical Industries, Roche; Travel/Accommodations/Meeting Expenses Unrelated to Activities Listed: Roche, Novartis, Teva Pharmaceutical Industries. Julien SavatovskyUNRELATED: Payment for Lectures Including Service on Speakers Bureaus: Medtronic, Sanofi, Biogen; Travel/Accommodations/Meeting Expenses Unrelated to Activities Listed: Bayer HealthCare, Philips Healthcare.

\section{REFERENCES}

1. Okuda DT, Mowry EM, Cree BA, et al. Asymptomatic spinal cord lesions predict disease progression in radiologically isolated syndrome. Neurology 2011;76:686-92 CrossRef Medline

2. Filippi M, Rocca MA, Ciccarelli O, et al; MAGNIMS Study Group. MRI criteria for the diagnosis of multiple sclerosis: MAGNIMS consensus guidelines. Lancet Neurol 2016;15:292-303 CrossRef Medline

3. Polman CH, Reingold SC, Banwell B, et al. Diagnostic criteria for multiple sclerosis: 2010 revisions to the McDonald criteria. Ann Neurol 2011;69:292-302 CrossRef Medline

4. Coret F, Bosca I, Landete Let al. Early diffuse demyelinating lesion in the cervical spinal cord predicts a worse prognosis in relapsingremitting multiple sclerosis. Mult Scler 2010;16, 935-41 CrossRef Medline

5. Rovira À, Wattjes MP, Tintoré M, et al. Evidence-based guidelines: MAGNIMS consensus guidelines on the use of MRI in multiple sclerosis-clinical implementation in the diagnostic process. Nat Rev Neurol 2015;11:471-82 CrossRef Medline

6. Vargas MI, Delavelle J, Kohler R, et al. Brain and spine MRI artifacts at 3 Tesla. J Neuroradiol 2009;36:74-81 CrossRef Medline

7. Bot JC, Barkhof F, Lycklama à Nijeholt GJ, et al. Comparison of a conventional cardiac-triggered dual spin-echo and a fast STIR sequence in detection of spinal cord lesions in multiple sclerosis. Eur Radiol 2000;10:753-58 CrossRef Medline

8. Taber KH, Herrick RC, Weathers SW, et al. Pitfalls and artifacts encountered in clinical MR imaging of the spine. Radiographics 1998;18:1499-1521 CrossRef Medline

9. McGowan J C. Technical issues for MRI examination of the spinal cord. J Neurol Sci 2000;172(Suppl 1):S27-31 CrossRef Medline

10. Chong AL, Chandra RV, Chuah KC, et al. Proton density MRI increases detection of cervical spinal cord multiple sclerosis lesions compared with T2-weighted fast spin-echo. AJNR Am J Neuroradiol 2016;37:180-84 CrossRef Medline

11. Thorpe JW, MacManus DG, Kendall BE, et al. Short tau inversion recovery fast spin-echo (fast STIR) imaging of the spinal cord in 
multiple sclerosis. Magn Reson Imaging 1994;12:983-89 CrossRef Medline

12. Nayak NB, Salah R, Huang JC, et al. A comparison of sagittal short $\mathrm{T} 1$ inversion recovery and $\mathrm{T} 2$-weighted FSE sequences for detection of multiple sclerosis spinal cord lesions. Acta Neurol Scand 2014;129: 198-203 CrossRef Medline

13. Poonawalla AH, Hou P, Nelson FA, et al. Cervical spinal cord lesions in multiple sclerosis: T1-weighted inversion-recovery MR imaging with phase-sensitive reconstruction. Radiology 2008;246:258-64 CrossRef Medline

14. White ML, Zhang Y, Healey K. Cervical spinal cord multiple sclerosis: evaluation with 2D multi-echo recombined gradient echo MR imaging. J Spinal Cord Med 2011;34:93-98 CrossRef Medline

15. Sundarakumar DK, Smith CM, Hwang WD, et al. Evaluation of focal cervical spinal cord lesions in multiple sclerosis: comparison of white matter-suppressed $\mathrm{T} 1$ inversion recovery sequence versus conventional STIR and proton density-weighted turbo spin-echo sequences. AJNR Am J Neuroradiol 2016;37:1561-66 CrossRef Medline

16. Riederer I, Karampinos DC, Settles M, et al. Double inversion recovery sequence of the cervical spinal cord in multiple sclerosis and related inflammatory diseases. AJNR Am J Neuroradiol 2015;36, 219-25 CrossRef Medline

17. Nair G, Absinta M, Reich DS. Optimized T1-MPRAGE sequence for better visualization of spinal cord multiple sclerosis lesions at $3 \mathrm{~T}$. AJNR Am J Neuroradiol 2013;34:2215-22 CrossRef Medline

18. Hou P, Hasan KM, Sitton CW, et al. Phase-sensitive T1 inversion recovery imaging: a time-efficient interleaved technique for improved tissue contrast in neuroimaging. AJNR Am J Neuroradiol 2005;26:1432-38 Medline

19. Vandenbroucke JP, von Elm E, Altman DG, et al; STROBE Initiative. Strengthening the Reporting of Observational Studies in Epidemiology (STROBE): explanation and elaboration. Int J Surg 2014;12: 1500-24 CrossRef Medline

20. Kurtzke JF. Rating neurologic impairment in multiple sclerosis: an expanded disability status scale (EDSS). Neurology 1983;33:1444-52 CrossRef Medline

21. Landis JR, Koch GG. An application of hierarchical kappa-type statistics in the assessment of majority agreement among multiple observers. Biometrics 1977;33:363-74 Medline

22. R Core Team. R: A language and environment for statistical computing. Vienna: R Foundation for Statistical Computing. 2014. http:// www.R-project.org/. Accessed 2016

23. Martin N, Malfair D, Zhao Y, et al. Comparison of MERGE and axial T2-weighted fast spin-echo sequences for detection of multiple sclerosis lesions in the cervical spinal cord. AJR Am J Roentgenol 2012;199:157-162 CrossRef Medline

24. Weier K1, Mazraeh J, Naegelin Y, et al. Biplanar MRI for the assessment of the spinal cord in multiple sclerosis. Mult Scler 2012;18: 1560-69 CrossRef Medline

25. Gass A, Rocca MA, Agosta F, et al; MAGNIMS Study Group. MRI monitoring of pathological changes in the spinal cord in patients with multiple sclerosis. Lancet Neurol 2015;14:443-54 CrossRef Medline

26. Alcaide-Leon P, Pauranik A, Alshafai L, et al. Comparison of sagittal FSE T2, STIR, and T1-weighted phase-sensitive inversion recovery in the detection of spinal cord lesions in MS at 3T. AJNR Am J Neuroradiol 2016;37:970-75 CrossRef Medline

27. Sombekke MH, Wattjes MP, Balk LJ, et al. Spinal cord lesions in patients with clinically isolated syndrome: a powerful tool in diagnosis and prognosis. Neurology 2013;80:69-75 CrossRef Medline

28. Traboulsee A, Simon JH, Stone L, et al. Revised Recommendations of the Consortium of MS Centers Task Force for a Standardized MRI Protocol and Clinical Guidelines for the Diagnosis and Follow-Up of Multiple Sclerosis. AJNR Am J Neuroradiol 2016;37:394-401 CrossRef Medline

29. Ramalho J, Castillo M, AlObaidy M, et al. High signal intensity in globus pallidus and dentate nucleus on unenhanced T1-weighted MR images: evaluation of two linear gadolinium-based contrast agents. Radiology 2015;276:836-44 CrossRef Medline

30. McDonald RJ, McDonald JS, Kallmes DF, et al. Intracranial gadolinium deposition after contrast-enhanced MR imaging. Radiology 2015;275:772-82 CrossRef Medline

31. Kanda $\mathrm{T}$, Osawa $\mathrm{M}$, Oba $\mathrm{H}$, et al. High signal intensity in dentate nucleus on unenhanced T1-weighted MR images: association with linear versus macrocyclic gadolinium chelate administration. $\mathrm{Ra}$ diology 2015;275:803-09 CrossRef Medline

32. Tartaglino LM, Friedman DP, Flanders AE, et al. Multiple sclerosis in the spinal cord: MR appearance and correlation with clinical parameters. Radiology 1995:195:725-32 CrossRef Medline

33. Curley M, Josey L, Lucas R, et al; Ausimmune Investigator Group. Adherence to MRI protocol consensus guidelines in multiple sclerosis: an Australian multi-centre study. J Med Imaging Radiat Oncol 2012;56:594-98 CrossRef Medline

34. Bot JC, Barkhof F, Polman CH, et al. Spinal cord abnormalities in recently diagnosed patients with MS: added value of spinal MRI examination. Neurology 2004;62:226-33 CrossRef Medline

35. Nijeholt GJ, Bergers E, Kamphorst W, et al. Post-mortem high-resolution MRI of the spinal cord in multiple sclerosis: a correlative study with conventional MRI, histopathology and clinical phenotype. Brain 2001;124:154-66 CrossRef Medline 